\title{
Electroproduction of a large invariant mass photon pair
}

\author{
A. Pedrak $\odot,{ }^{1}$ B. Pire $\odot,{ }^{2}$ L. Szymanowski®,$^{1}$ and J. Wagner ${ }^{1}$ \\ ${ }^{1}$ National Centre for Nuclear Research (NCBJ), Pasteura 7, 02-093 Warsaw, Poland \\ ${ }^{2}$ Centre de Physique Théorique, CNRS, École Polytechnique, I. P. Paris, 91128 Palaiseau, France
}

(Received 7 March 2020; accepted 4 June 2020; published 25 June 2020)

\begin{abstract}
We study the exclusive electroproduction of a photon pair in the kinematical regime where the diphoton invariant mass is large and where the nucleon flies almost in its original direction. We discuss the relative importance of the QCD process where the two photons originate from a quark line compared to the single (double) Bethe-Heitler processes where one (two) photons originate from the lepton line. This process turns out to be a promising tool to study generalized parton distributions in the nucleon, both at the medium energy of JLab and at a high energy electron ion collider.
\end{abstract}

DOI: 10.1103/PhysRevD.101.114027

\section{INTRODUCTION}

Hard electroproduction processes are a powerful probe of the hadronic structure. Their understanding in the framework of the QCD collinear factorization of hard amplitudes in specific kinematics in terms of generalized parton distributions (GPDs) and hard perturbatively calculable coefficient functions [1,2] offers a way to access the inner structure of nucleons and light nuclei.

In a previous work $[3,4]$, we studied the exclusive photoproduction of two photons on an unpolarized proton or neutron target,

$\gamma(q, \epsilon)+N\left(P_{1}, s_{1}\right) \rightarrow \gamma\left(k_{1}, \epsilon_{1}\right)+\gamma\left(k_{2}, \epsilon_{2}\right)+N^{\prime}\left(P_{2}, s_{2}\right)$,

in the kinematical regime of large invariant diphoton squared mass $M_{\gamma \gamma}^{2}=\left(k_{1}+k_{2}\right)^{2}$ of the final photon pair and small momentum transfer $t=\left(P_{2}-P_{1}\right)^{2}$ between the initial and the final nucleons. This process shares many features with timelike Compton scattering (TCS), the photoproduction of a large mass lepton pair [5]. We enlarge our study to the more general case of electroproduction,

$$
\begin{aligned}
& e(k, \lambda)+N\left(P_{1}, s_{1}\right) \rightarrow e^{\prime}\left(k^{\prime}, \lambda\right)+\gamma\left(k_{1}, \epsilon_{1}\right) \\
& \quad+\gamma\left(k_{2}, \epsilon_{2}\right)+N^{\prime}\left(P_{2}, s_{2}\right),
\end{aligned}
$$

Published by the American Physical Society under the terms of the Creative Commons Attribution 4.0 International license. Further distribution of this work must maintain attribution to the author(s) and the published article's title, journal citation, and DOI. Funded by SCOAP . which generalizes the real photoproduction process (1) to the virtual photon case

$\gamma^{*}(q, \epsilon)+N\left(P_{1}, s_{1}\right) \rightarrow \gamma\left(k_{1}, \epsilon_{1}\right)+\gamma\left(k_{2}, \epsilon_{2}\right)+N^{\prime}\left(P_{2}, s_{2}\right)$,

with $q=k-k^{\prime}$ and $q^{2} \neq 0$. As will be demonstrated below, this reaction has a number of interesting features, both for moderate energy in the JLab domain and for the high energy domain of a future electron-ion collider (EIC) [6], and can be used as an important source of information for future programs aiming at the extraction of GPDs [7]. There are three processes contributing to the reaction (2), namely, the QCD process of Fig. 1 where the two photons are emitted from a quark, the single Bethe-Heitler process of Fig. 2 where one photon is emitted from a lepton and the other photon from a quark, and the double Bethe-Heitler process of Fig. 3 where the two photons originate from a lepton. As demonstrated below, their relative importance depends very much on the kinematical conditions, and particularly on the value of $Q^{2}=-\left(k^{\prime}-k\right)^{2}$.

The plan of this paper is the following. In Sec. II, we review the kinematics of the reaction. Section III presents the calculation of the amplitudes of the three processes which contribute and shows the main features of their contributions to the differential cross sections. In Sec. IV, we compare their relative contributions to the differential cross section from medium (JLab) to high (EIC) energies and examine to which extent the quasireal electroproduction of a large mass diphoton can be described by the photoproduction cross section with the known flux of Weizsäcker-Williams equivalent photons. Section V gathers some conclusions. The Appendix presents the spinor techniques used in our calculations. 


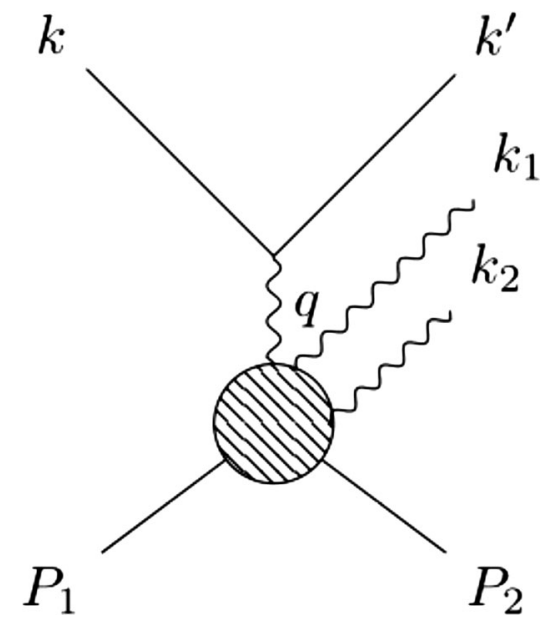

FIG. 1. The QCD process contributing to $e N \rightarrow e^{\prime} \gamma \gamma N^{\prime}$.

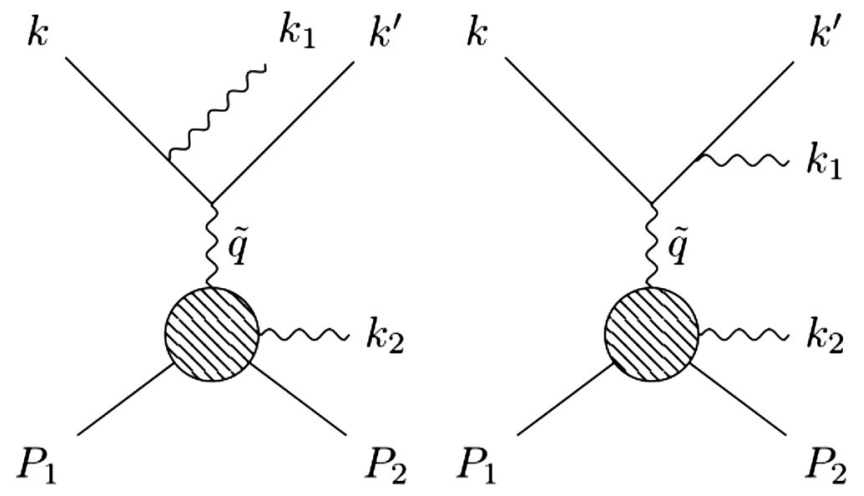

FIG. 2. The single Bethe-Heitler process contributing to $e N \rightarrow e^{\prime} \gamma \gamma N^{\prime}$. Two other graphs with $k_{1} \leftrightarrow k_{2}$ interchange are not shown.

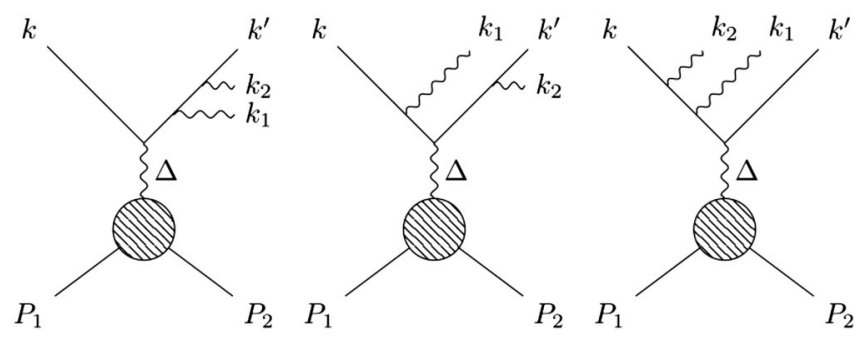

FIG. 3. The double Bethe-Heitler process contributing to $e N \rightarrow e^{\prime} \gamma \gamma N^{\prime}$. Three other graphs with $k_{1} \leftrightarrow k_{2}$ interchange are not shown.

\section{KINEMATICS}

Let us first present the kinematics of the process (2). It is most similar to the one of double deeply virtual Compton scattering (DDVCS) [8]. We decompose every momenta on a Sudakov basis as

$$
v^{\mu}=a n^{\mu}+b p^{\mu}+v_{\perp}^{\mu},
$$

with $p$ and $n$ the light-cone vectors

$$
\begin{aligned}
p^{\mu} & =\frac{\sqrt{s}}{2}(1,0,0,1), \quad n^{\mu}=\frac{\sqrt{s}}{2}(1,0,0,-1), \\
p \cdot n & =\frac{s}{2},
\end{aligned}
$$

and

$$
v^{+}=v \cdot n, \quad v_{\perp}^{\mu}=\left(0, v^{x}, v^{y}, 0\right), \quad v_{\perp}^{2}=-\vec{v}_{t}^{2} .
$$

The particle momenta, in the chosen reference frame, read

$$
\begin{aligned}
k^{\mu} & =|\vec{k}|(1, \sin \theta \cos \phi, \sin \theta \sin \phi, \cos \theta) \\
& =\frac{|\vec{k}|}{\sqrt{s}}\left[(1+\cos \theta) p^{\mu}+(1-\cos \theta) n^{\mu}\right]+k_{\perp}^{\mu},
\end{aligned}
$$

$$
\begin{aligned}
& q^{\mu}=y n^{\mu}-\frac{Q^{2}}{y s} p^{\mu}, \quad k^{\prime \mu}=k^{\mu}-q^{\mu}, \\
& P_{1}^{\mu}=(1+\xi) p^{\mu}+\frac{M^{2}}{s(1+\xi)} n^{\mu}, \\
& P_{2}^{\mu}=(1-\xi) p^{\mu}+\frac{M^{2}+\vec{\Delta}_{t}^{2}}{s(1-\xi)} n^{\mu}+\Delta_{\perp}^{\mu},
\end{aligned}
$$

$k_{1}^{\mu}=\alpha_{1} n^{\mu}+\frac{\left(\vec{\kappa}_{t}-\vec{\Delta}_{t} / 2\right)^{2}}{\alpha_{1} s} p^{\mu}+\kappa_{\perp}^{\mu}-\frac{\Delta_{\perp}^{\mu}}{2}$,

$k_{2}^{\mu}=\alpha_{2} n^{\mu}+\frac{\left(\vec{\kappa}_{t}+\vec{\Delta}_{t} / 2\right)^{2}}{\alpha_{2} s} p^{\mu}-\kappa_{\perp}^{\mu}-\frac{\Delta_{\perp}^{\mu}}{2}$,

where $M$ is the mass of the nucleon and $\xi$ is the skewness parameter. We define the momentum transfer $\Delta^{\mu}=$ $P_{2}^{\mu}-P_{1}^{\mu}$. In the $\gamma \gamma$ center-of-mass system, we define the angles $\theta_{\gamma \gamma}, \phi_{\gamma \gamma}$ as the polar and azimuthal angles of $\gamma\left(k_{1}\right)$. Since the azimuthal dependence of the process can only depend on the difference $\phi_{\gamma \gamma}-\phi$, we fix, by convention, $\phi=0$.

The total center-of-mass energy squared of the electronnucleon system $S_{e N}$ is, neglecting terms of order $M^{2} / S_{e N}$ or $t / S_{e N}$,

$$
S_{e N} \approx(1+\xi) s,
$$

and the skewness parameter equals

$$
\xi \approx \frac{Q^{2}+M_{\gamma \gamma}^{2}}{2 y S_{e N}-Q^{2}-M_{\gamma \gamma}^{2}} .
$$

Since here we enlarge our previous study of the photoproduction process, we define the large (factorization) scale as 


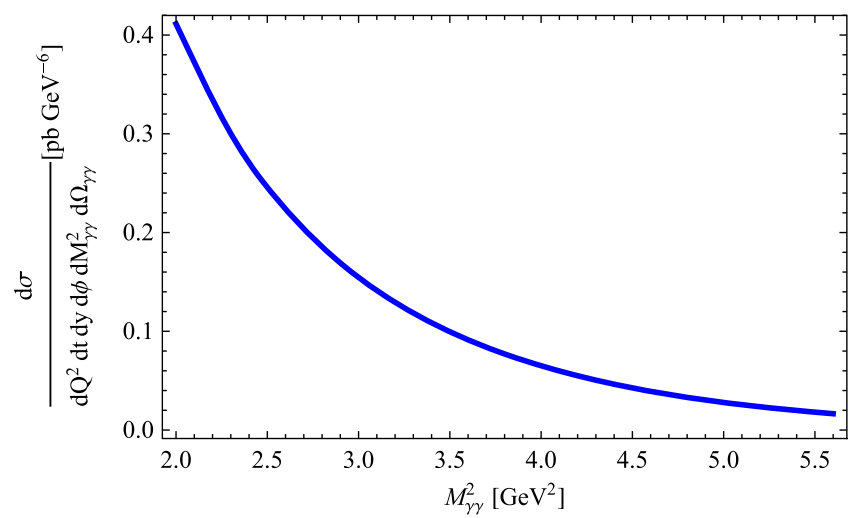

FIG. 4. The $M_{\gamma \gamma}^{2}-$ dependence of the QCD process contribution to the $e N \rightarrow e^{\prime} \gamma \gamma N^{\prime}$ differential cross section, at $Q^{2}=$ $10^{-3} \mathrm{GeV}^{2}, s=20 \mathrm{GeV}^{2}, y=0.8, \theta_{\gamma \gamma}=3 \pi / 8, \phi_{\gamma \gamma}=0$.

$$
M_{\gamma \gamma}^{2}=\left(k_{1}+k_{2}\right)^{2}=\frac{4 \vec{\kappa}_{t}^{2}}{\sin ^{2} \theta_{\gamma \gamma}},
$$

which, as usual, is quite arbitrary but sufficient for a leading order computation; $t=\Delta^{2}$ is kept small with respect to this scale. To sum up the kinematical domain we are interested in, we choose

$$
\begin{aligned}
& M_{\gamma \gamma}^{2} \gg \Lambda_{\mathrm{QCD}}^{2} \\
& y S_{e N} \gg \Lambda_{\mathrm{QCD}}^{2}, \\
& \frac{M_{\gamma \gamma}^{2}}{y S_{e N}}=O(1), \\
& \xi=O(1) .
\end{aligned}
$$

We will write the differential cross section of the process as

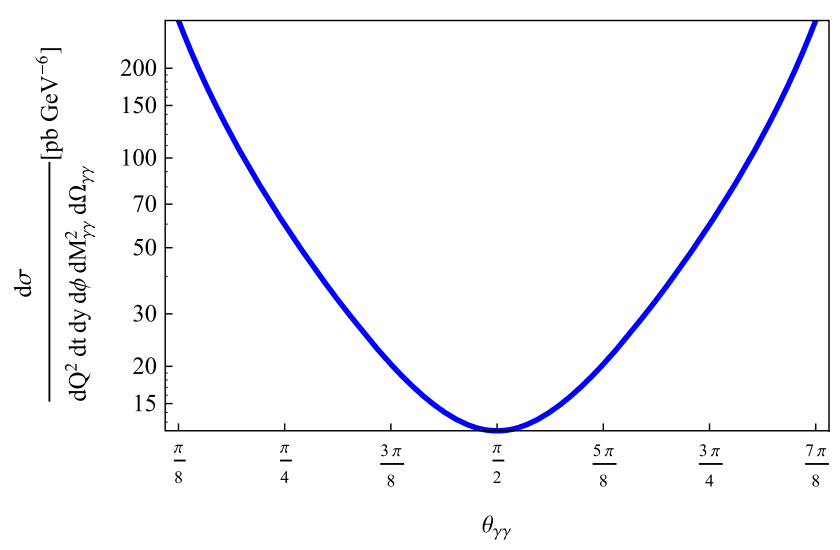

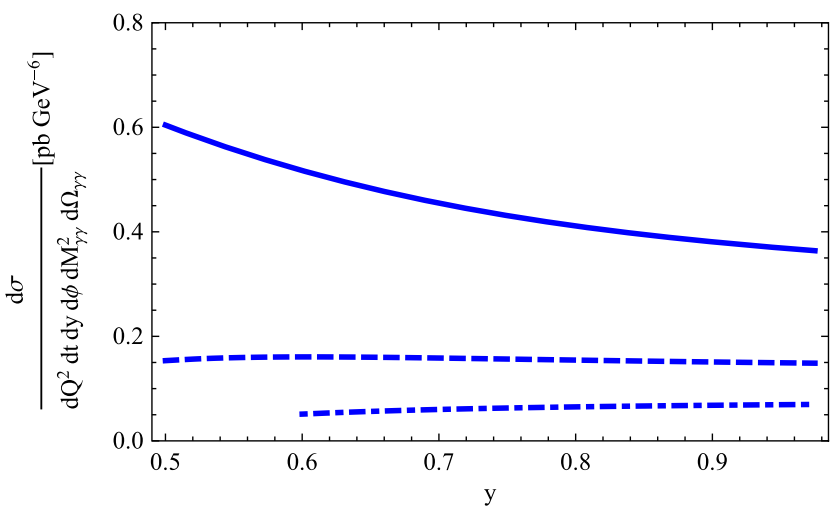

FIG. 6. The $y$ dependence of the QCD process contribution to the $e N \rightarrow e^{\prime} \gamma \gamma N^{\prime}$ differential cross section, at $Q^{2}=10^{-3} \mathrm{GeV}^{2}$, $s=20 \mathrm{GeV}^{2}$ and $M_{\gamma \gamma}^{2}=2 \mathrm{GeV}^{2}$ (full curve), $M_{\gamma \gamma}^{2}=3 \mathrm{GeV}^{2}$ (dashed curve) and $M_{\gamma \gamma}^{2}=4 \mathrm{GeV}^{2}$ (dash-dotted curve), at $\theta_{\gamma \gamma}=$ $3 \pi / 8, \phi_{\gamma \gamma}=0$.

$\frac{d \sigma}{d Q^{2} d y d t d \phi d M_{\gamma \gamma}^{2} d \Omega_{\gamma \gamma}}=\frac{1}{2} \frac{\alpha_{e m}^{4}}{16(2 \pi)^{3}} \frac{1}{\left(S_{e N}-M^{2}\right)^{2}}|\mathcal{M}|^{2}$.

Momentum conservation puts a lower value to $y$ since $y S_{e N}$ must be larger than $M_{\gamma \gamma}^{2}$. For JLab energies, this $y_{\min }$ is not small.

\section{THE PRODUCTION PROCESSES}

We choose to calculate, at leading order in $\alpha_{e m}$ and $\alpha_{S}$, the amplitudes of the three contributing processes following the Kleiss-Sterling spinor techniques [9], with the external photon polarization vectors in the gauge defined by the $p$ vector. Useful formulas are gathered in the Appendix. To our knowledge, this is the first time that this formalism is used for a hard exclusive process. We neglect the lepton mass everywhere.

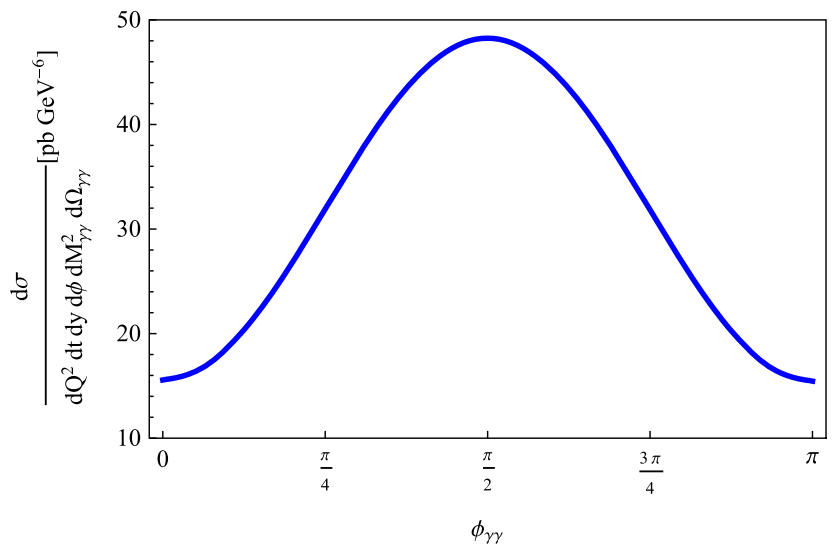

FIG. 5. The angular dependence (in the $\gamma \gamma$ center-of-mass system) of the QCD process contribution to the $e N \rightarrow e^{\prime} \gamma \gamma N^{\prime}$ cross section, at $s=20 \mathrm{GeV}^{2}, y=0.6, M_{\gamma \gamma}^{2}=3 \mathrm{GeV}^{2}$ and $Q^{2}=10^{-5} \mathrm{GeV}^{2}$, as a function of $\theta_{\gamma \gamma}$ for $\phi_{\gamma \gamma}=\pi / 8$ (left panel) and as a function of $\phi_{\gamma \gamma}$ for $\theta_{\gamma \gamma}=3 \pi / 8$ (right panel). 


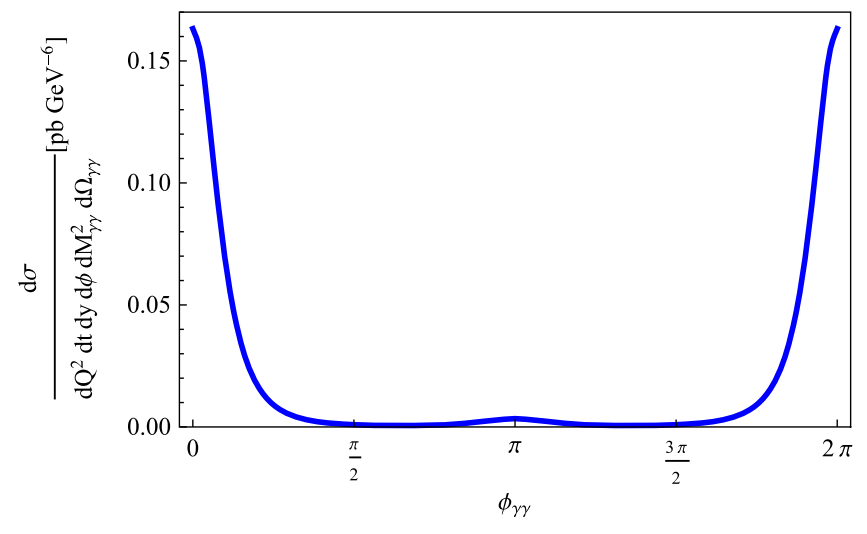

FIG. 7. The azimuthal dependence of the single BetheHeitler process contribution to the $e N \rightarrow e^{\prime} \gamma \gamma N^{\prime}$ cross section, at $s=20 \mathrm{GeV}^{2}, Q^{2}=1 \mathrm{GeV}^{2}, \quad y=0.6, \quad \theta_{\gamma \gamma}=3 \pi / 8$ and $M_{\gamma \gamma}^{2}=3 \mathrm{GeV}^{2}$.

\section{A. The QCD process}

Calculating the amplitude of the QCD process follows the same lines as in the photoproduction case [3]. We detail in the Appendix the case of the helicity amplitude $(++\rightarrow++-+)$. Other cases are similar.

The simplified kinematical relations used to calculate the hard coefficient functions are

$$
\begin{aligned}
& \alpha_{1}+\alpha_{2}=y, \quad \alpha_{1}=\frac{y}{2}\left[1+\sqrt{1-\frac{4 \vec{\kappa}_{t}^{2}}{2 \xi s y-Q^{2}}}\right], \\
&|\vec{k}|=\frac{\sqrt{s}}{2}\left[1+\frac{Q^{2}}{s} \frac{1-y}{y^{2}}\right], \quad \cos \theta=-\frac{1-\frac{Q^{2}}{s} \frac{1-y}{y^{2}}}{1+\frac{Q^{2}}{s} \frac{1-y}{y^{2}}} .
\end{aligned}
$$

Because of the charge conjugation properties of the $\left(\gamma^{*}, \gamma_{1}, \gamma_{2}\right)$ system, the QCD process turns out to be sensitive only to the nonsinglet combination of GPDs:

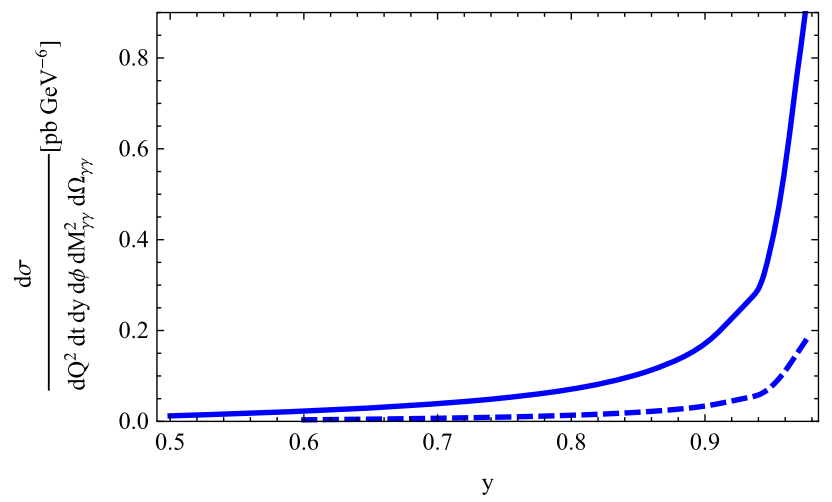

$$
H^{(-)}(x)=\sum_{q} Q_{q}^{3}\left(H^{q}(x)+H^{q}(-x)\right)
$$

There is no contribution from the singlet quark, nor gluonic GPDs, at any order of the QCD calculation.

Let us now present some features of the contribution of the QCD process to the unpolarized differential cross section $\frac{d \sigma^{e N \rightarrow e^{\prime} \gamma \gamma N^{\prime}}}{d Q^{2} d d y d d M_{\gamma \gamma}^{2} d \Omega_{\gamma \gamma}}$ at $t=t_{\min }$ (i.e., for $\Delta_{T}=0$ ), $s=20 \mathrm{GeV}^{2}$. For numerical estimates we used the Goloskokov-Kroll model [10] for GPD $H$, neglecting all other contributions (which were responsible for less than $1 \%$ of the cross section in the photoproduction case). Since, for very small values of $Q^{2}$, this cross section scales like $1 / Q^{2}$, as it should following the Weizsäcker-Williams formula, the results for different small $Q^{2}$ values than presented on the plots can easily be deduced. The $M_{\gamma \gamma}^{2}$ dependence of the differential cross sections, shown in Fig. 4 for $Q^{2}=10^{-3} \mathrm{GeV}^{2}$, follows an effective powerlike behavior $1 / M_{\gamma \gamma}^{2 n}$ with $n \approx 3$. We show in Fig. 5 the $\gamma \gamma$ angular dependence in the $\gamma \gamma$ center-of-mass system. The range in $\theta_{\gamma \gamma}$ is limited due to the requirement that the factorization scale for the single Bethe-Heitler process is high enough-as discussed in the Sec. III B. The $y$ dependence shown in Fig. 6 is quite weak (provided $y>y_{\min }$ ). The magnitude of the cross section indicates that the discussed process is accessible in the currently running and designed experiments at JLAB and EIC.

\section{B. The single Bethe-Heitler process}

Some care is needed to apply the collinear factorization framework to calculate the QCD part of the single BetheHeitler amplitude of Fig. 2. Indeed, the photon entering the hadronic part must be hard enough to justify a QCD description of the virtual Compton scattering subprocess $\gamma^{*}(\tilde{q}) N \rightarrow \gamma N^{\prime}$. Since

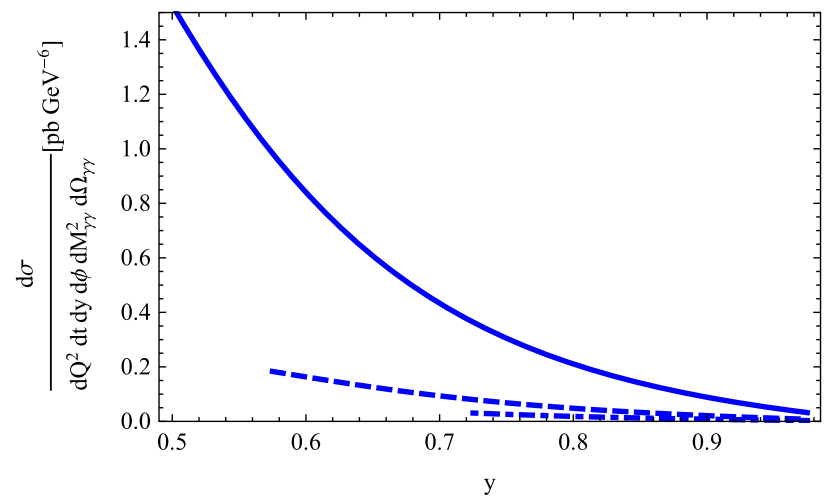

FIG. 8. The $y$ dependence of the single Bethe-Heitler process contribution to the $e N \rightarrow e^{\prime} \gamma \gamma N^{\prime}$ cross section, at $s=20 \mathrm{GeV}^{2}$. Left panel: $Q^{2}=10^{-3} \mathrm{GeV}^{2}$ and $M_{\gamma \gamma}^{2}=3 \mathrm{GeV}^{2}$ (solid curve) and $M_{\gamma \gamma}^{2}=4 \mathrm{GeV}^{2}$ (dashed curve). Right panel: $Q^{2}=1 \mathrm{GeV}^{2}$ and $M_{\gamma \gamma}^{2}=$ $2 \mathrm{GeV}^{2}$ (solid curve), $M_{\gamma \gamma}^{2}=3 \mathrm{GeV}^{2}$ (dashed curve), and $M_{\gamma \gamma}^{2}=4 \mathrm{GeV}^{2}$ (dash-dotted curve). 

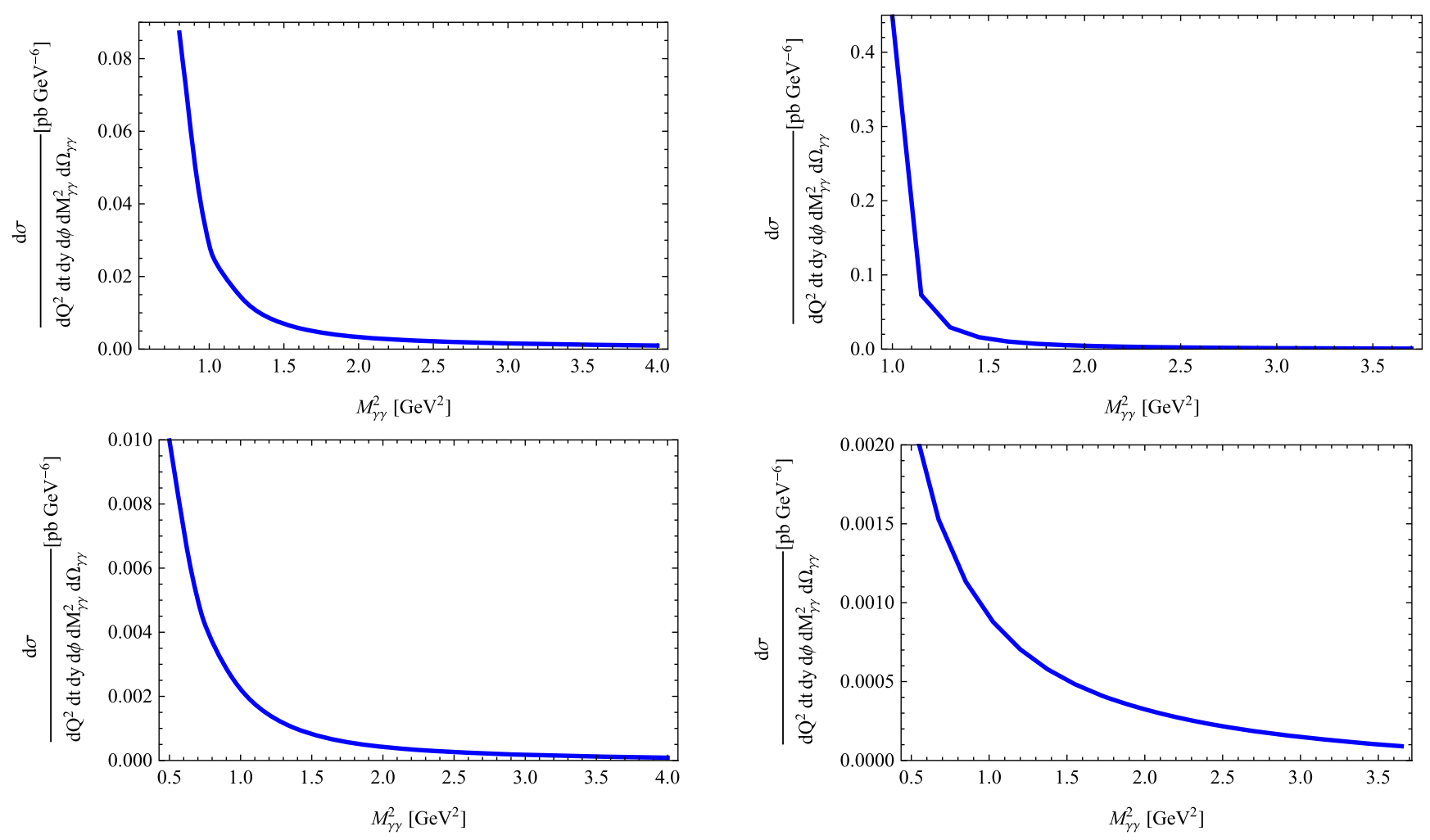

FIG. 9. $\quad M_{\gamma \gamma}^{2}$ dependence of the contribution of the double Bethe-Heitler process to the differential cross section at $\theta_{\gamma \gamma}=3 \pi / 8, y=0.8$, for $\phi_{\gamma \gamma}=0$ (first line) or $\phi_{\gamma \gamma}=\pi / 2$ (second line), and for $Q^{2}=1$ (resp. 2) $\mathrm{GeV}^{2}$ on the left (resp. right) panel.

$$
\tilde{q}=q-k_{1} \quad \text { or } \quad \tilde{q}=q-k_{2},
$$

one should distinguish the case where $-\tilde{q}^{2}$ is large enough to apply QCD factorization, and the converse case where a description in terms of nucleon polarizabilities [11] is more appropriate. In practice, we shall only consider the kinematics which prevent $-\tilde{q}^{2}$ from getting smaller than $1 \mathrm{GeV}^{2}$, which is assured by choosing diphoton squared mass above $3 \mathrm{GeV}^{2}$.

One should properly define the kinematics so that the factorized formalism can be straightforwardly applied. This implies defining a new Sudakov basis where the $\gamma^{*}(\tilde{q}) N\left(P_{1}\right)$ define the $(+,-)$ basis. We choose to do so with

$\tilde{p}^{\mu}=p^{\mu} ; \quad \tilde{n}^{\mu}=n^{\mu}-\frac{\kappa_{\perp}^{2}}{2 n p} p^{\mu}+\kappa_{\perp}^{\mu} \quad$ when $\tilde{q}=q-k_{1}$,

$\tilde{p}^{\mu}=p^{\mu} ; \quad \tilde{n}^{\mu}=n^{\mu}-\frac{\kappa_{\perp}^{2}}{2 n p} p^{\mu}-\kappa_{\perp}^{\mu} \quad$ when $\tilde{q}=q-k_{2}$,

so that $\tilde{p} \cdot \tilde{n}=p \cdot n$. The hard part is independent of these variations on the Sudakov vector $n$. Then we write the hadronic part of the single Bethe-Heitler amplitude with the replacement
$\frac{H^{q}(x)}{s} \bar{U}\left(P_{2}, s_{2}\right) \not \varkappa U\left(P_{1}, s_{1}\right) \rightarrow \frac{H^{q}(x)}{s} \bar{U}\left(P_{2}, s_{2}\right) \tilde{h} U\left(P_{1}, s_{1}\right)$,

for the contribution of the $H^{q}(x, \xi, t) \mathrm{GPD}$, and similar terms for the contributions of other GPDs. ${ }^{1}$

As the usual DVCS amplitude, because of the charge conjugation properties of the $\left(\gamma^{*}, \gamma\right)$ system, the single Bethe-Heitler process turns out to be sensitive to the singlet combination of GPDs,

$$
H^{(+)}(x)=\sum_{q} Q_{q}^{2}\left(H^{q}(x)-H^{q}(-x)\right)
$$

and (at higher order in $\alpha_{s}$ ) will benefit from gluonic GPD contributions. NLO QCD corrections calculated for DVCS [13] can straightforwardly be applied to this process.

\footnotetext{
${ }^{1}$ Let us remark that we encounter here a theoretical uncertainty related to the choice of the lightlike vector $n^{\mu}$ spanning the longitudinal subspace, which results in the appearance of kinematical ambiguities of predictions usually attributed to higher twists effects. As discussed in [12], the predictions for a leading twist- 2 contribution to the scattering amplitude are sensitive to the choice of $n^{\mu}$ appearing in the factorization formula and in the parametrization of momenta. The full analysis of kinematical ambiguities in our process along the lines of Ref. [12] is obviously beyond the scope of our paper.
} 
The $\phi_{\gamma \gamma}$ dependence of the single Bethe-Heitler process contribution is plotted in Fig. 7. We show in Fig. 8 the $y$ dependence of the single Bethe-Heitler process contribution to the cross section, for both very small and sizable $Q^{2}$ (there is no curve for the $M_{\gamma \gamma}^{2}=2 \mathrm{GeV}^{2}$ on the left plot, as the factorization scale is too small in that case for a description in terms of GPD).

\section{The double Bethe-Heitler process}

As the Bethe-Heitler contribution to the DVCS amplitude, the double Bethe-Heitler contribution is expressed in terms of the known Dirac and Pauli electromagnetic form factors $F_{1}\left(\Delta^{2}\right)$ and $F_{2}\left(\Delta^{2}\right)$ as

$$
\begin{aligned}
\mathcal{M}_{\mathrm{BH}}= & \frac{-1}{\Delta^{2}} \bar{u}\left(k^{\prime}, \lambda\right) \mathcal{L}^{\mu} u(k, \lambda) \bar{U}\left(P_{2}, s_{2}\right) \\
& \times\left[\gamma^{\mu} F_{1}\left(\Delta^{2}\right)+\sigma^{\mu \nu} \frac{i \Delta^{\nu}}{2 M} F_{2}\left(\Delta^{2}\right)\right] U\left(P_{1}, s_{1}\right),
\end{aligned}
$$

with
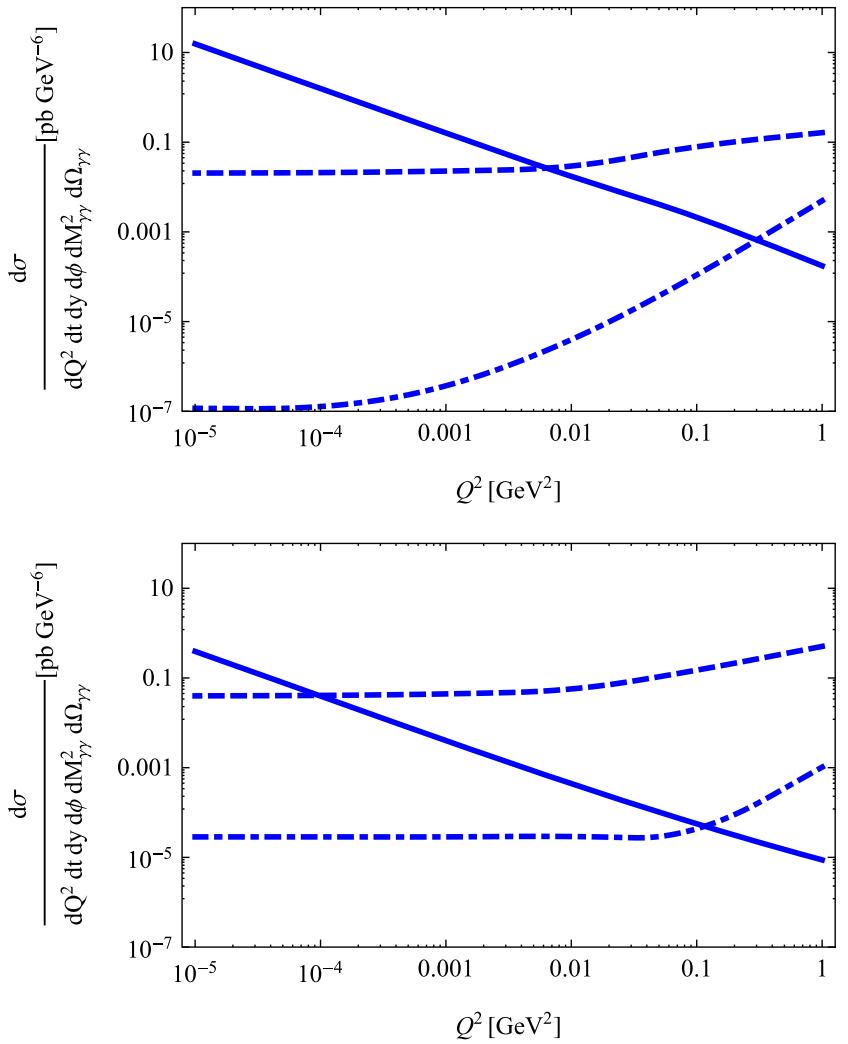

$$
\begin{aligned}
\mathcal{L}^{\mu}= & \gamma^{\mu} \frac{1}{\hat{k}^{\prime}+\hat{\Delta}+i \epsilon} \hat{\epsilon}_{1} \frac{1}{\hat{k}^{\prime}+\hat{\Delta}+\hat{k}_{1}+i \epsilon} \hat{\epsilon}_{2} \\
& +\hat{\epsilon}_{1} \frac{1}{\hat{k}^{\prime}+\hat{k}_{1}+i \epsilon} \gamma^{\mu} \frac{1}{\hat{k}^{\prime}+\hat{\Delta}+\hat{k}_{1}+i \epsilon} \hat{\epsilon}_{2} \\
& +\hat{\epsilon}_{1} \frac{1}{\hat{k}^{\prime}+\hat{k}_{1}+i \epsilon} \hat{\epsilon}_{2} \frac{1}{\hat{k}^{\prime}+\hat{k}_{2}+\hat{k}_{1}+i \epsilon} \gamma^{\mu} \\
& +\left(k_{1} \leftrightarrow k_{2}\right) .
\end{aligned}
$$

We calculate all the helicity amplitudes following the spinor techniques briefly described in the Appendix.

For illustration, we show in Fig. 9 the $M_{\gamma \gamma}^{2}$ dependence of the contribution of the double Bethe-Heitler process to the differential cross section. Since our calculation is valid without any restriction on kinematics, we show the differential cross section even for rather small values of $M_{\gamma \gamma}^{2}$. This contribution decreases quite quickly with $M_{\gamma \gamma}$ and is more sizable when $\phi_{\gamma \gamma}$ is small.

\section{COMPARISON OF THE PROCESSES}

The relative importance of the different processes contributing to $e N \rightarrow e^{\prime} \gamma \gamma N^{\prime}$ is illustrated in Fig. 10 where we
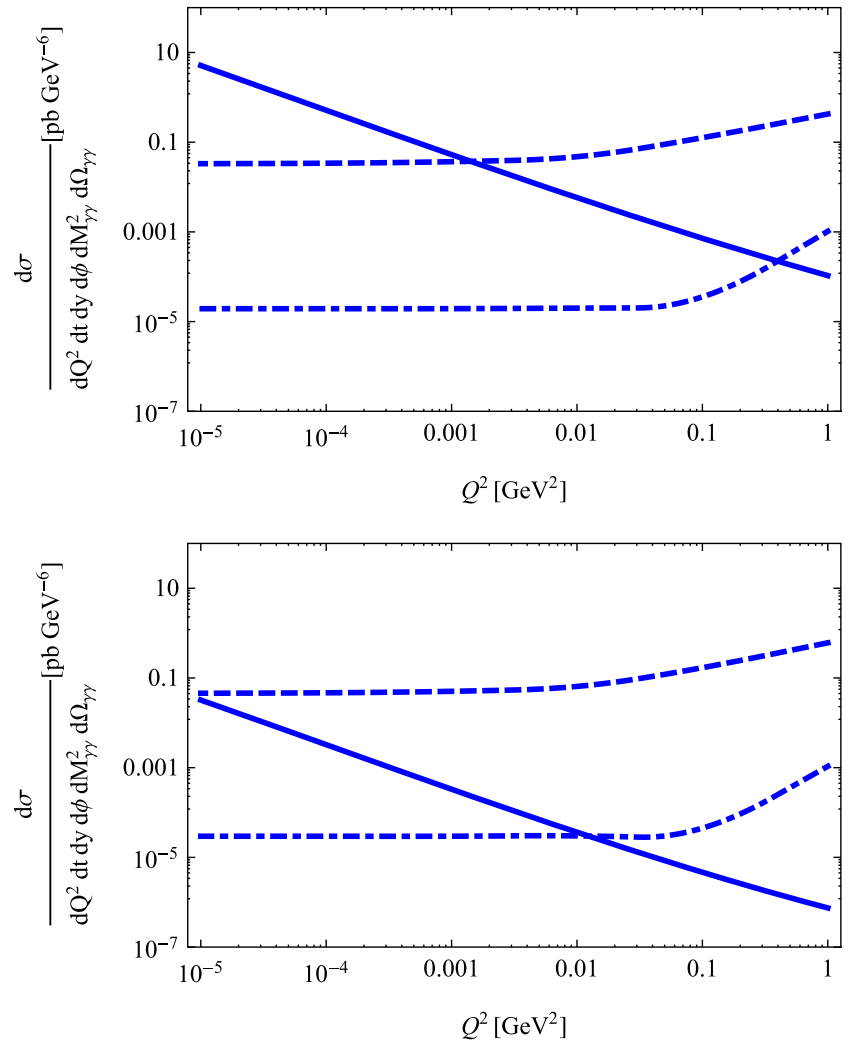

FIG. 10. The relative importance of the different processes contributing to $e N \rightarrow e^{\prime} \gamma \gamma N^{\prime}$-shown here (from left to right and from top to bottom) for $s=20 \mathrm{GeV}^{2}, s=100 \mathrm{GeV}^{2}, s=1000 \mathrm{GeV}^{2}$ and $s=10000 \mathrm{GeV}^{2}$ at the kinematical point $M_{\gamma \gamma}^{2}=3 \mathrm{GeV}^{2}$, $\theta_{\gamma \gamma}=3 \pi / 8, \phi_{\gamma \gamma}=0, y=0.6$ - depends much on the value of $Q^{2}$. The QCD process (solid curve) dominates at very low $Q^{2}$, the single Bethe-Heitler process (dashed curve) dominates at higher $Q^{2}$, while the double Bethe-Heitler process (dotted curve) is always subdominant. 
plot the contributions of the three processes (neglecting their interferences) at a characteristic kinematical point. Their magnitude indeed depends much on the value of $Q^{2}$. The QCD process (solid curve) dominates at very low $Q^{2}$, the single Bethe-Heitler process (dashed curve) dominates at higher $Q^{2}$, while the double Bethe-Heitler process (dotted curve) is always much smaller than either the QCD or the single Bethe-Heitler process in the kinematical range we are interested in; we can thus neglect this latter contribution for any phenomenological purpose.

In the quasireal photoproduction limit, we recover the results of our previous work [3]: the C-odd (valence) GPDs are accessed in a peculiar way since the QCD amplitude is proportional to these GPDs taken at their border values $x= \pm \xi$. By scrutinizing the $Q^{2}$ dependence of the single Bethe-Heitler and the QCD amplitudes, we can quantify what we mean by "quasireal," which turns out to depend very much on the overall energy domain. One may conclude that one can safely apply the WeizsäckerWilliams equivalent photon approximation $[14,15]$ for $Q^{2}<10^{-3} \mathrm{GeV}^{2}$ at JLab but only for $Q^{2}<10^{-5} \mathrm{GeV}^{2}$ at EIC.

\section{CONCLUSION}

Our calculation of the leading order leading twist amplitude of reaction (2) has demonstrated that the electroproduction of a large invariant mass diphoton is an interesting process to analyze in the collinear factorization framework, both at current experimental facilities such as JLab and Compass at CERN, but also in future high energy experiments. The amplitude has very specific properties which should be very useful for future GPD extraction programs e.g., [7].

One may sum up our results as follows:

(i) On the one hand, the QCD process dominates the amplitude in the very small $Q^{2}$ domain for JLab energies. We quantified the maximal value of $Q^{2}$ for the Weizsäcker-Williams approximation to be valid. The conclusions written in our previous work on the photoproduction process then apply to an electroproduction experiment. For completeness, let us remind the reader that the main conclusion of this study is that the amplitude only depends on C-odd GPDs at the border values $x= \pm \xi$.

(ii) On the other hand, the single Bethe-Heitler process strongly dominates the amplitude in the domain where $Q^{2}$ is not extremely small, especially in the large energy domain of the EIC. We can then apply collinear QCD factorization to the amplitude provided that the virtuality of the exchanged photon is large enough, and we verified that this was indeed the case for large enough values of $M_{\gamma \gamma}^{2}$, typically above $3 \mathrm{GeV}^{2}$. In this window, the diphoton electroproduction coalesces to a deeply virtual Compton scattering but with the interesting difference thatbecause of the smallness of the double Bethe-Heitler amplitude-it is not polluted anymore by the BetheHeitler process which usually dominates the QCD process. This opens a new window for the extraction of C-even GPDs, and in particular for gluon GPDs which have been shown to give a large contribution in next to leading order (NLO) $O\left(\alpha_{S}\right)$ calculations [13]. This NLO study is however out of the scope of the present paper.

Although very important for the nucleon tomography program [16], we did not detail the dependence on $\Delta_{\perp}$ which enters through the $t$ dependence of GPDs. Nor did we enter the discussion of higher twist effects, which should be taken into account as much as possible as in the DVCS case [12,17]. Finally, let us stress that NLO QCD corrections are likely to be important and should definitely be computed before a sensible phenomenology of our process is undertaken. Together with a further hint of the factorization of our process, it should yield interesting information on the analytic structure of these QCD corrections in a process where both timelike $M_{\gamma \gamma}^{2}$ and spacelike $Q^{2}$ scales coexist, in contradistinction with the DVCS vs TCS cases $[18,19]$.

\section{ACKNOWLEDGMENTS}

We acknowledge useful conversations with Cédric Lorcé. The work of J.W. is supported by Grant No. 2017/26/M/ST2/01074 of the National Science Center in Poland, whereas the work of L. S. is supported by Grant No. 2019/33/B/ST2/02588 of the National Science Center in Poland. This project is also co-financed by the Polish-French collaboration agreement Polonium, by the Polish National Agency for Academic Exchange and COPIN-IN2P3, and by the European Union's Horizon 2020 Research and Innovation Programme under Grant Agreement No. 824093.

\section{APPENDIX: HELICITY AMPLITUDES IN THE SPINOR TECHNIQUES}

We are considering the amplitude for the QCD process contribution to the electroproduction of two photons:

$$
\begin{aligned}
& e(k, \mu)+N\left(P_{1}, s_{1}\right) \rightarrow e\left(k^{\prime}, \mu\right)+N\left(P_{2}, s_{2}\right) \\
& \quad+\gamma\left(k_{1}, \lambda_{1}\right)+\gamma\left(k_{2}, \lambda_{2}\right)
\end{aligned}
$$

assuming particular helicities and spin combination $\mu=$ $\mu^{\prime}=+; s_{1}=s_{2}=+, ; \lambda_{1}=+, \lambda_{2}=-$.

We neglect the electron mass, which ensures helicity conservation along the electron line. Following [9], we write the massive spinor momenta as the sum of two lightlike momenta (here in the simplest case of $\Delta_{T}=0$ ): 


$$
\begin{aligned}
& P_{1}=p_{1}+p_{2} ; \quad P_{2}=p_{1}^{\prime}+p_{2}^{\prime} ; \\
& p_{2}=(1+\xi) p ; \quad p_{1}=\frac{M^{2}}{1+\xi} n ; \\
& p_{2}^{\prime}=(1-\xi) p ; \quad p_{1}^{\prime}=\frac{M^{2}}{1-\xi} n,
\end{aligned}
$$

which allows us to decompose the nucleon spinors as a linear combination of two massless fermion spinors:

$$
\begin{aligned}
& U\left(P_{1},+\right)=\frac{s\left(p_{1}, p_{2}\right)}{M} u_{+}\left(p_{1}\right)+u_{-}\left(p_{2}\right) ; \\
& U\left(P_{1},-\right)=\frac{t\left(p_{1}, p_{2}\right)}{M} u_{-}\left(p_{1}\right)+u_{+}\left(p_{2}\right),
\end{aligned}
$$

with the $s\left(p_{1}, p_{2}\right)=-t^{*}\left(p_{1}, p_{2}\right)$ products defined as

$s\left(p_{1}, p_{2}\right)=\left(p_{1}^{y}+i p_{1}^{z}\right) \sqrt{\frac{p_{2}^{0}-p_{2}^{x}}{p_{1}^{0}-p_{1}^{x}}}-\left(p_{2}^{y}+i p_{2}^{z}\right) \sqrt{\frac{p_{1}^{0}-p_{1}^{x}}{p_{2}^{0}-p_{2}^{x}}}$,

so that $s\left(p_{1}, p_{2}\right) s^{*}\left(p_{1}, p_{2}\right)=t\left(p_{1}, p_{2}\right) t^{*}\left(p_{1}, p_{2}\right)=2 p_{1} \cdot p_{2}$. The photon polarization vectors in the $p$ gauge read

$$
\epsilon_{ \pm}^{\mu}\left(k_{i}\right)=N_{i} \bar{u}_{ \pm}\left(k_{i}\right) \gamma^{\mu} u_{ \pm}(p) N_{i}=\frac{1}{2 \sqrt{k_{i} \cdot p}} .
$$

The amplitude is written as the product of the leptonic and hadronic parts,

$$
i \mathcal{M}=\frac{i}{Q^{2}} L^{\alpha} H_{\alpha}
$$

where the leptonic part reads

$$
L^{\alpha}=\bar{u}_{+}\left(k^{\prime}\right) \gamma^{\alpha} u_{+}(k),
$$

and the hadronic part reads, focusing on the contribution of the $H(x, \xi, t)$ GPD,

$$
H_{\alpha}=\sum_{q} \frac{Q_{q}^{3}}{2} \int d x \operatorname{Tr}\left[\tilde{\mathcal{M}}_{\alpha}(x) \not p\right] F_{++}^{q}(x),
$$

with $Q_{u}=2 / 3, Q_{d}=-1 / 3, \ldots$ and

$$
\begin{aligned}
F_{++}^{q}(x)= & \frac{H^{q}(x)}{s} \bar{U}_{+}\left(P_{2}\right) \not h U_{+}\left(P_{1}\right) \\
= & \frac{H^{q}(x)}{s}\left[\frac{t\left(p_{2}^{\prime}, p_{1}^{\prime}\right) s\left(p_{1}, p_{2}\right)}{M^{2}} s\left(p_{1}^{\prime}, n\right) t\left(n, p_{1}\right)\right. \\
& \left.+t\left(p_{2}^{\prime}, n\right) s\left(n, p_{2}\right)\right],
\end{aligned}
$$

(a)

(b)

(c)

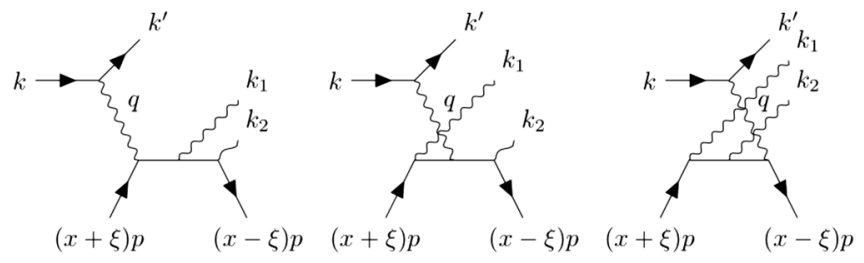

FIG. 11. The Feynman diagrams contributing to the QCD process; the other 3 diagrams are obtained by $k_{1} \leftrightarrow k_{2}$ interchange.

The three diagrams of Fig. 11 give the following contributions:

$\tilde{\mathcal{M}}_{\alpha}^{(a)}=\phi_{-}^{*}\left(k_{2}\right)\left[\frac{(x-\xi) \not p+\not k_{2}}{D_{1}(x)}\right] \phi_{+}^{*}\left(k_{1}\right)\left[\frac{(x-\xi) \not p+\not k_{1}+\not k_{2}}{D_{2}(x)}\right] \gamma_{\alpha}$,

$\tilde{\mathcal{M}}_{\alpha}^{(b)}=\phi_{-}^{*}\left(k_{2}\right)\left[\frac{(x-\xi) \not x+\not k_{2}}{D_{1}(x)}\right] \gamma_{\alpha}\left[\frac{(x+\xi) \not p-\not k_{1}}{D_{3}(-x)}\right] \phi_{+}^{*}\left(k_{1}\right)$,

$\tilde{\mathcal{M}}_{\alpha}^{(c)}=\gamma_{\alpha}\left[\frac{(x+\xi) \not p-\not k_{1}-\not k_{2}}{D_{2}(-x)}\right] \phi_{-}^{*}\left(k_{2}\right)\left[\frac{(x+\xi) \not p-\not \not_{1}}{D_{3}(-x)}\right] \phi_{+}^{*}\left(k_{1}\right)$,

with the propagator denominators

$D_{1}(x)=\alpha_{2} s(x-\xi+i \varepsilon) \equiv \alpha_{2} s D(x, \xi)$,

$D_{2}(x)=y s\left(x+\xi-\frac{Q^{2}}{s y}+i \varepsilon\right)=y s D\left(x,-\xi^{\prime}\right)$,

$D_{3}(x)=\alpha_{1} s(x-\xi+i \varepsilon)=\alpha_{1} s D(x, \xi)$,

with $D(x, \xi)=x-\xi+i \varepsilon$ and where we denote $\xi^{\prime}=$ $\xi-\frac{Q^{2}}{s y}$. Denoting

$\mathcal{A}^{(x)}=\operatorname{Tr}\left[\tilde{\mathcal{M}}_{\alpha}^{(x)} \not p\right] L^{\alpha} ; \quad{ }^{c} \mathcal{A}^{(x)}=\operatorname{Tr}\left[\tilde{\mathcal{M}}_{\alpha}^{(x)}\left(k_{1} \leftrightarrow k_{2}\right) \not p\right] L^{\alpha}$,

we get 


$$
\begin{aligned}
\mathcal{A}^{(b)}+{ }^{c} \mathcal{A}^{(b)}= & 8 N_{1} N_{2} t\left(k, k_{1}\right) s\left(k_{2}, k^{\prime}\right)\left[\frac{1}{D(x, \xi) D(x,-\xi)^{*}}+(x \rightarrow-x)\right] ; \\
\mathcal{A}^{(a)}+{ }^{c} \mathcal{A}^{(a)}+\mathcal{A}^{(c)}+{ }^{c} \mathcal{A}^{(c)}= & \frac{8 N_{1} N_{2}}{y s}\left(\left(s\left(p, k_{2}\right) t\left(k_{1}, p\right) t(k, p) s\left(p, k^{\prime}\right)+t\left(p, k_{1}\right) s\left(k_{2}, p\right) s\left(k^{\prime}, p\right) t(p, k)\right)\right. \\
& \times\left[\frac{1}{D\left(x,-\xi^{\prime}\right)}+(x \rightarrow-x)\right]+\left(s\left(p, k_{2}\right) t\left(k_{1}, k_{2}\right) t(k, p) s\left(k_{2}, k^{\prime}\right)\right. \\
& \left.\left.+t\left(p, k_{1}\right) s\left(k_{2}, k_{1}\right) s\left(k^{\prime}, p\right) t\left(k_{1}, k\right)\right)\left[\frac{1}{D(x, \xi) D\left(x,-\xi^{\prime}\right)}+(x \rightarrow-x)\right]\right) .
\end{aligned}
$$

Denoting the C-odd charge weighted GPD combination as

$$
H^{(-)}(x)=\sum_{q} Q_{q}^{3}\left(H^{q}(x)+H^{q}(-x)\right),
$$

we need the following integrals:

$$
\begin{gathered}
I(\xi)=\int_{-1}^{1} \frac{H^{(-)}(x)}{x-\xi+i \varepsilon} d x=\int_{-1}^{1} \frac{H^{(-)}(x)-H^{(-)}(\xi)}{x-\xi} d x+H^{(-)}(\xi) \log \frac{1-\xi}{1+\xi}-i \pi H^{(-)}(\xi), \\
\sum_{q} Q_{q}^{3} \int_{-1}^{1}\left[\frac{1}{D(x, \xi) D(x,-\xi)^{*}}+(x \rightarrow-x)\right] H^{q}(x) d x=\frac{1}{2 \xi}\left(I(\xi)-I^{*}(-\xi)\right), \\
\sum_{q} Q_{q}^{3} \int_{-1}^{1}\left[\frac{1}{D\left(x,-\xi^{\prime}\right)}+(x \rightarrow-x)\right] H^{q}(x) d x=\frac{1}{2}\left(I\left(-\xi^{\prime}\right)-I^{*}\left(\xi^{\prime}\right)\right), \\
\sum_{q} Q_{q}^{3} \int_{-1}^{1}\left[\frac{1}{D(x, \xi) D\left(x,-\xi^{\prime}\right)}+(x \rightarrow-x)\right] H^{q}(x)=\frac{1}{2\left(\xi+\xi^{\prime}\right)}\left(I(\xi)-I^{q}\left(-\xi^{\prime}\right)-I^{*}(-\xi)+I^{*}\left(\xi^{\prime}\right)\right),
\end{gathered}
$$

which we evaluate numerically with the GPD parametrizations of Ref. [10].

The resulting expression for the helicity amplitude is then given by

$$
\begin{aligned}
\mathcal{M}= & \frac{1}{Q^{2}} \frac{1}{2 s}\left[\frac{t\left(p_{2}^{\prime}, p_{1}^{\prime}\right) s\left(p_{1}, p_{2}\right)}{M^{2}} s\left(p_{1}^{\prime}, n\right) t\left(n, p_{1}\right)+t\left(p_{2}^{\prime}, n\right) s\left(n, p_{2}\right)\right] 8 N_{1} N_{2}\left\{t\left(k, k_{1}\right) s\left(k_{2}, k^{\prime}\right) \frac{1}{2 \xi}\left[I(\xi)-I^{*}(-\xi)\right]\right. \\
& +\left(s\left(p, k_{2}\right) t\left(k_{1}, p\right) t(k, p) s\left(p, k^{\prime}\right)+t\left(p, k_{1}\right) s\left(k_{2}, p\right) s\left(k^{\prime}, p\right) t(p, k)\right) \frac{1}{2 s y}\left[I\left(-\xi^{\prime}\right)-I^{*}\left(\xi^{\prime}\right)\right] \\
& \left.+\left(s\left(p, k_{2}\right) t\left(k_{1}, k_{2}\right) t(k, p) s\left(k_{2}, k^{\prime}\right)+t\left(p, k_{1}\right) s\left(k_{2}, k_{1}\right) s\left(k^{\prime}, p\right) t\left(k_{1}, k\right)\right) \frac{1}{2 s y} \frac{1}{\xi+\xi^{\prime}}\left[I(\xi)-I^{q}\left(-\xi^{\prime}\right)-I^{*}(-\xi)+I^{*}\left(\xi^{\prime}\right)\right]\right\} .
\end{aligned}
$$

[1] D. Müller, D. Robaschik, B. Geyer, F.-M. Dittes, and J. Hořejši, Fortschr. Phys. 42, 101 (1994); X. Ji, Phys. Rev. Lett. 78, 610 (1997); A. V. Radyushkin, Phys. Rev. D 56, 5524 (1997); J. C. Collins and A. Freund, Phys. Rev. D 59, 074009 (1999).
[2] M. Diehl, Phys. Rep. 388, 41 (2003); A. V. Belitsky and A. V. Radyushkin, Phys. Rep. 418, 1 (2005); S. Boffi and B. Pasquini, Riv. Nuovo Cimento 30, 387 (2007).

[3] A. Pedrak, B. Pire, L. Szymanowski, and J. Wagner, Phys. Rev. D 96, 074008 (2017); 100, 039901(E) (2019). 
[4] A. Pedrak, B. Pire, L. Szymanowski, and J. Wagner, Proc. Sci., DIS2019 (2019) 196.

[5] E. R. Berger, M. Diehl, and B. Pire, Eur. Phys. J. C 23, 675 (2002); M. Boër, M. Guidal, and M. Vanderhaeghen, Eur. Phys. J. A 51, 103 (2015); I. V. Anikin et al., Acta Phys. Pol. B 49, 741 (2018); B. Pire, L. Szymanowski, and S. Wallon, Phys. Rev. D 101, 074005 (2020).

[6] A. Accardi et al., Eur. Phys. J. A 52, 268 (2016); J. L. Abelleira Fernandez et al. (LHeC Study Group), J. Phys. G 39, 075001 (2012).

[7] B. Berthou et al., Eur. Phys. J. C 78, 478 (2018); H. Moutarde, P. Sznajder, and J. Wagner, Eur. Phys. J. C 78, 890 (2018); H. Moutarde, P. Sznajder, and J. Wagner, Eur. Phys. J. C 79, 614 (2019).

[8] M. Guidal and M. Vanderhaeghen, Phys. Rev. Lett. 90, 012001 (2003); A. V. Belitsky and D. Mueller, Phys. Rev. Lett. 90, 022001 (2003); B. Z. Kopeliovich, I. Schmidt, and M. Siddikov, Phys. Rev. D 82, 014017 (2010).

[9] R. Kleiss and W. J. Stirling, Nucl. Phys. B262, 235 (1985).

[10] S. V. Goloskokov and P. Kroll, Eur. Phys. J. C 53, 367 (2008); P. Kroll, H. Moutarde, and F. Sabatié, Eur. Phys. J. C 73, 2278 (2013).

[11] H. Fonvieille, B. Pasquini, and N. Sparveris, arXiv:1910 .11071 .
[12] V. M. Braun and A. N. Manashov, Phys. Rev. Lett. 107, 202001 (2011); V. M. Braun, A. N. Manashov, and B. Pirnay, Phys. Rev. Lett. 109, 242001 (2012); V. M. Braun, A. N. Manashov, D. Müller, and B. M. Pirnay, Phys. Rev. D 89, 074022 (2014).

[13] B. Pire, L. Szymanowski, and J. Wagner, Phys. Rev. D 83, 034009 (2011); Few Body Syst. 53, 125 (2012); H. Moutarde, B. Pire, F. Sabatie, L. Szymanowski, and J. Wagner, Phys. Rev. D 87, 054029 (2013).

[14] P. Kessler, Acta Phys. Austriaca 41, 141 (1975).

[15] S. Frixione, M. L. Mangano, P. Nason, and G. Ridolfi, Phys. Lett. B 319, 339 (1993).

[16] M. Burkardt, Phys. Rev. D 62, 071503 (2000); 66, 119903 (E) (2002); Eur. Phys. J. C 25, 223 (2002); 31, 277(E) (2003); J. P. Ralston and B. Pire, Phys. Rev. D 66, 111501 (2002).

[17] I. V. Anikin, B. Pire, and O. V. Teryaev, Phys. Rev. D 62 , 071501 (2000); A. V. Belitsky and D. Mueller, Nucl. Phys. B589, 611 (2000).

[18] D. Mueller, B. Pire, L. Szymanowski, and J. Wagner, Phys. Rev. D 86, 031502 (2012).

[19] O. Grocholski, H. Moutarde, B. Pire, P. Sznajder, and J. Wagner, Eur. Phys. J. C 80, 171 (2020). 